\title{
UNIFIED CELLULAR NEURAL NETWORK CELL DYNAMICAL EQUATION USING DELTA OPERATOR
}

\author{
HARI C. REDDY ${ }^{1,3} \&$ GEORGE S. MOSCHYTZ 2 \\ 'Department of Electrical Engineering, California State University, Long Beach, CA, USA. \\ 'Institute of Signal and Information Processing, ETH, Zurich, Switzerland. \\ ${ }^{3}$ Department of Electrical and Computer Engineering, University of California, Irvine, CA, USA.
}

\begin{abstract}
The signal processing algorithms based on conventional shift operator tend to be ill-conditioned in situations involving fast sampling and shorter wordlength. To alleviate this problem delta operator based analysis and design has been proposed for high speed digital signal processing and control systems. The advantage for delta ( $\delta$ ) operator seems to come from the fact as sampling period $T_{1} \rightarrow 0$, the discrete time system process resembles that of continuous time system. In this paper we develop a unified cellular neural network (CNN) cell model using the delta operator approach. The model gives a general discrete-time (DT) CNN cell dynamics in which the sampling period $T_{2}$ is an explicit parameter. As $T_{3} \rightarrow 0$, we get the continuous time (CT)-CNN equation. Several results connected with the stability and robustness of CT-CNN and DT-CNN can be linked using this approach. This approach highlights the similarities, rather than the differences between discrete and continuous CNNs, thus allowing continuous insights to be applied to the discrete CNN case. Further, more importantly from the implementation point of view delta operator based DT-CNN cell design can be obtained using $\delta^{-1}$ as an integrator $\left\{\right.$ instead of a delay $\left.\left(\mathrm{z}^{-1}\right)\right\}$. The $\delta^{-1}$ integrator can be realized using switched current/switched capacitor circuits. The dynamic circuit element in the DT - CNN is thus " $\delta^{-1 "}$.
\end{abstract}

\section{INTRODUCTION}

Due mainly to the pioneering contributions and exposition by Middleton and Goodwin [1-3], there has been significant interest in delta operator formulated discrete-time systems. When analog signals are sampled at high speeds (high sampling rates) and processed by sampled-data or digital filters, the delta operator approach for the design yields filters with low sensitivity, low noise and higher dynamic range than using shift operator approach. Signal processing algorithms tend to be ill conditioned at high sampling rates when formulated using shift (q)-operator . In the design of circuits and systems for processing discrete-time signals that arise from the sampling of an underlying continuous process, the limiting behavior of the circuits as the sampling rate increases is seldom considered. The advantage for delta operator based design appears to be due to the fact as the sampling period $T_{3} \rightarrow 0$, the discrete-time system resembles the underlying continuous system process. So, it is desirable to reformulate the design under high sampling rate situations with $\delta$-operator instead of standard q-operator. It has also been shown that appropriate $\delta$-operator based numerical schemes can reduce finite wordlength effects in digital simulation of nonlinear dynamic systems with fine grid and/or short wordlength situations. Coefficient sensitivity of solution orbit for fixed point and floating point implementations have been investigated recently $[4,5]$. When CT-nonlinear system is simulated with fine grid size (or, under fast sampling), it has been proven that a solution orbit computed via delta $\delta$-scheme is less sensitive to coefficient perturbation compared to shift (q)-operator scheme. Thus, it has been established that in high sampling rate cases the delta operator approach is superior to shift operator in both linear and nonlinear signal processing/control systems. This provides us with the motivation to study the dynamics of CNN in terms of delta operator.

Cellular neural networks (CNNs), proposed by Chua and Yang in 1988 [6] consist of arrays of elementary processing units called cells. Each cell is connected to a set of nearby cells (neighbors). CNNs are well suited for image processing applications in view of their two-dimensional structures and local interconnections which are typical characteristics of image processing algorithms. The other applications are in pattern recognition and motion detection etc. The computational paradigm of $\mathrm{CNN}$ which constitutes a class of locally connected nonlinear system is very suitable for VLSI implementation. Continuous time (CT)-CNNs are nonlinear dynamic systems described by differential equations. Discrete time (DT)-CNN proposed in [7] with advantages similar to CT-CNN (in terms of local activity etc.) is described by nonlinear finite difference equation.

This paper deals with the formulation of unified cell dynamics for CNN using delta operator. It is shown that when the discrete-time signal is obtained by sampling an underlying continuous time process the $\mathrm{CNN}$ dynamics 
corresponding to the discrete signal case converges in well defined sense to the solution of the underlying continuous $\mathrm{CNN}$ dynamics as the sampling period approaches zero.

The following is the layout of the paper. In section II we describe briefly the delta operator and in the next section we study the delta operator formulation of the CNN cell state equation. In section IV we summarize some of the salient benefits of the unifying model.

\section{DELTA $(\delta)$ OPERATOR}

Let the discrete-time sequence generated by sampling a continuous time signal $x(t)$ be $x\left(k T_{3}\right)$ where $T_{2}$ is the sampling period and $k=0,1,2, \ldots$ We assume that $T_{s}=0$ signifies $x\left(k T_{s}\right)=x(t)$. For $T_{2} \neq 0$ we denote $x\left(k T_{2}\right)=x(n), n=0,1,2, \ldots$

The delta $(\delta)$ operator is defined as:

$$
\begin{aligned}
\delta\left[x\left(k T_{s}\right)\right] & =\frac{x\left(k T_{s}+T_{s}\right)-x\left(k T_{s}\right)}{T_{s}}, T_{s} \neq 0 \\
& =\frac{q\left[x\left(k T_{s}\right)\right]-x\left(k T_{s}\right)}{T_{s}}, T_{s} \neq 0
\end{aligned}
$$

In the above $q$ is a forward shift operator. The $q$ and $\delta$ (also called forward Euler operator) are related as:

$$
\begin{aligned}
\delta & \equiv \frac{q-1}{T_{s}} \\
& =a \cdot q+b \\
(a & \left.=-b=1 / T_{s}\right)
\end{aligned}
$$

Thus, the delta operator can be considered as one obtained as a linear combination of shift operator. In view of this $\delta$ as a dynamic operator provides the same flexibility and implementability as shift operator ( one can formulate and solve the state equations with almost same ease as with $q$ operator [1] ). Further, it provides the continuity when $T_{a}=0$. Referring to equation ( 1 ) we have

$$
\lim _{T, \rightarrow 0} \delta\left[x\left(k T_{s}\right)\right]=\frac{d}{d t} x(t)
$$

It is thus a discrete time analog of the differentiation operator.

As explained in [5], two interpretations of $T_{3}$ in equation (1) could be given: For digital signal processing/ sampled data systems in processing of analog signals, $T$, represents the sampling (switching) period with "time" units. Here, $T_{2} \rightarrow 0$, results in an (underlying) analog systems. If the system is inherently discrete, $\delta$ which is obtained as a linear combination of " $q$ " operator has an extra design parameter $T_{3}$. This can be used in achieving better analysis and design of systems [1].

In view of all this it is advantageous to formulate discrete time (DT) CNN cell state equation using the delta operator.

\section{DELTA OPERATOR BASED DT-CNN CELL EQUATION ( $\delta$ DT-CNN)}

CNN is a collection of identical cells or processing units connected only to their nearest neighbors. These are arranged regularly on a two dimensional array. Each cell denoted by index c, is excited by a set of nearby cells located within a distance $r$ in the cell grid. Let $N_{r}(c)$ be the cell $r$-neighborhood. This neighborhood consists of cell $c$ and its interacting neighborhood cells. We consider uniform CNN where all the inner cells are identical. The cell state given by $x_{c}\left(k T_{3}\right)$ provides cell energy information as a function of time; the cell output $y_{d}\left(k T_{3}\right)$ is related to cell state through a nonlinearity which is a piecewiselinear function; the cell input $u$ along with the initial cell state for $k=0$ represents the external excitation of the cell. With this we now define delta operator formulated DT. CNN cell equation as follows:

$$
\begin{aligned}
& \begin{aligned}
\tau \cdot \delta\left[x_{c}\left(k T_{s}\right)\right]= & -x_{c}\left(k T_{s}\right)+\sum_{d \in N_{r}(c)} A_{c d} \cdot y_{d}\left(k T_{s}\right) \\
& +\sum_{d \in N_{r}(c)} B_{c d} U_{d}+I_{c}
\end{aligned} \\
& \text { or } \tau \cdot \delta\left[x_{c}\left(k T_{s}\right)\right]=-x_{c}\left(k T_{s}\right)+A \cdot y+B \cdot U+I \\
& y_{d}\left(k T_{s}\right)=f\left[x_{c}\left(k T_{s}\right)\right]
\end{aligned}
$$

$\tau$ is a constant denoting cell time constant. In the above A, B are feedback and control matrices and I represents overall network bias. The CNN operates by a proper choice of template set $\mathrm{A}, \mathrm{B}$, and I and by appropriately assigning the initial data, say the image to be processed, to either input $u$ or the initial state $x(0)$. The desired output is obtained as a stable equilibrium solution of the system described above.

As sampling period $T_{s} \rightarrow 0, \quad x\left(k T_{s}\right)$ denotes the continuous time $(C T)$ signal $x(t)$ and $\delta=d / d t$. This yields CT-CNN cell dynamical equation given by Chua and Yang [6 ]. 


$$
\begin{aligned}
\tau \cdot \frac{d}{d t}\left[x_{c}(t)\right]= & -x_{c}(t)+\sum_{d \in N_{r}(c)} A_{c d} \cdot y_{d}(t) \\
& +\sum_{d \in N_{r}(c)} B_{c d} U_{d}+I_{c}
\end{aligned}
$$

$y_{d}(t)=f\left[x_{c}(t)\right]$

The block diagram for eqn. (3) is shown in Fig. 1. It is to be observed that $\delta^{-1}$ block represents a causal operation. $\delta^{-1}$ can be realized by a switched capacitor or switched current circuits with the same ease as the $q$ (delay) operator [8]. CT-CNN model can be derived from Fig. 1 with $\delta^{-1}$ replaced by a CT- integrator for $T_{2}=0$. The main point here is that the discrete time $\mathrm{CNN}$ can be realized with $\delta^{-1}$ as a dynamic element instead of the delay $\mathrm{z}^{-1}$ as a dynamic element. By so doing we have discrete time dynamics in the circuit that smoothly tends towards continuous time CNN as switching frequency in the sampled data circuits is made high. In other words analog sampled data CNN circuit in the limit becomes analog continuous time CNN. Thus, all the benefits of CTCNN are propagated to DT-CNN through this approach. We cannot establish such a correspondence with the present q-operator based DT-CNN model.

If we expand equation (3) using equation (1) we get the qoperator based DT-CNN cell difference equation. (with $\left.x\left(k T_{s}\right)=x(n)\right)$

$$
\begin{aligned}
x_{c}(n+1)= & -\left(\frac{T_{s}-\tau}{\tau}\right) x_{c}(n)+\frac{T_{s}}{\tau} \sum_{d \in N_{r}(c)-} A_{c d} \cdot y_{d}(n) \\
& +\frac{T_{s}}{\tau} \sum_{d \in N_{,}(c)} B_{c d} U_{d}+\frac{T_{s}}{\tau} \cdot I_{c}
\end{aligned}
$$

$$
y_{d}(n)=f\left[x_{c}(n)\right]
$$

From the above we can obtain the DT-CNN model given by Harrer and Nossek [7] with $\tau=T_{3}$. The equation (5) is the general form of DT-CNN. In the next section we briefly discuss how the stability and robust template design of continuous and discrete time CNNs are related using the delta model.

\section{WHAT ARE THE MERITS OF THE DELTA} OPERATOR APPROACH?

1. The operator unifies CT-CNN and DT-CNN cell equations.
2. $\delta^{-1}$ as a causal operator can be realized as a discrete integrator using switched capacitor and/or switched current techniques.

3. It has been shown that for high sampling rates the $\delta^{-1}$ based realizations in linear $\delta$-operator based discrete time filters provides better finite word length(sensitivity) properties . It is expected that the low sensitivity property is carried to switched current and switched capacitor sampled data circuits (involving a nonlinear element) such as a CNN cell. The $\delta^{-1}$ based cell design may thus yield a robust cell structure. This is a problem for further investigation

4. In view of the smooth convergence to the continuous case, the stability results of the CT$\mathrm{CNN}$ and $\delta$ DT-CNN can be related. We would like to state the following result:

Result 1:If the discrete time CNN formulated using the delta operator (as in equation 3 ) is stable then the resulting continuous time CNN ( eqn. 4 ) is also stable. Alternatively, the necessary condition for $\delta$ DT-CNN to be stable is that the resulting CT-CNN cell equation is stable.

It has been shown that $T_{3}<2 \tau$ (where $T_{3}$ is the switching period and $\tau$ is the cell time constant) ensures the stability for many sampled data CNN Image processing applications. The Result 1 is in fact a limiting condition of $T_{3}<2 \tau$.

5. Robust template design for CT- CNN has been investigated recently [9]. Circuit implementation of $\mathrm{CNN}$ entails some physical limitations. A robust $\mathrm{CNN}$ is defined as one which operates as desired even when subjected to implementation inaccuracies. In [ 9 ] a robust $\mathrm{CNN}$ means a set of parameters $A, B, I$ for which any perturbation of the form $(A+\Delta A), \quad(B+\Delta B), \quad(I+\Delta D)$, with $\Delta \mathrm{A}, \Delta \mathrm{B}, \Delta \mathrm{I}$ containing the fractional errors of the parameters, leads to the same output as the unperturbed set. It would be interesting to develop the robust template design for delta operator formulated discrete time cell equation. This would give another result (similar to the above stability result) connecting the continuous and discrete time cell dynamics.

Result 2: If certain A, B and I values provide a robust design for delta discrete time CNN, then 
the same values would ensure robustness for the underlying continuous time $\mathrm{CNN}$. The robustness in CT-CNN provides only a necessary condition for $\delta \mathrm{DT}-\mathrm{CNN}$.

The conclusions listed in (4 and 5) cannot be obtained using the shift operator (q).

\section{CONCLUSIONS}

The main purpose of this paper has been to motivate the use of $\delta^{-1}$ as a dynamic causal operator (integrator) in the formulation of CNN cell equation and the cell circuit design. Much work ( both theoretical and experimental) need to be done to show that this is a better alternative to shift operator in the design of DT-CNN for high speed image processing and other applications. What we have done is to provide an unity to cell dynamics for analog continuous and sampled data CNN. Some of the advantages of so doing are discussed. The work of Middleton and Goodwin and others on the use of delta operator for high speed digital signal processing and control provides us the necessary impetus to expand it to the CNN paradigm.

\section{Acknowledgement:}

The authors would like to express their sincere appreciation to Professor Leon Chua for his kind comments and discussions. Also, the first author wishes to express his thanks to Mr. Baharam Mirzai of ETH-Z for discussions regarding $\mathrm{CNN}$ theory and Robust Design. Also, thanks are due to Mr. I. Hung Khoo for his help in the preparation of the manuscript and discussions.

\section{REFERENCES}

[1] G. C. Goodwin, R. H. Middleton and H. V. Poor, "High-speed digital signal processing and control", Proc. of the IEEE, vol 80 (2), pp 240-259, Feb 1992.

[2] R. H. Middleton and G. C. Goodwin, "Improved finite word length characteristics in digital control using delta operators", IEEE Transactions on Automatic Control, vol 36, pp 314-321, Mar 1986.

[3] R. H. Middleton and G. C. Goodwin, Digital Control and Estimation: $A$ Unified Approach, Prentice-Hall, 1990.

[4] M.M. Ekanayake and K. Premaratne, "Twodimensional delta-operator formulated discrete-time systems: analysis and synthesis of minimum roundoff noise realizations", Proc. IEEE-ISCAS, Atlanta, May, 1996.

[5] K. Premaratne and P.H. Bauer, "Delta-operator formulated discrete-time systems", Proc. IEEE-ISCAS, Atlanta, May, 1996.

[6] L. O. Chua and L. Yang, "Cellular neural networks: Theory," IEEE Trans. Circuits Syst., vol. 35, pp 1257. 1272, 1988.

[7] H. Harrer and J. A. Nossek, "Discrete-time cellular neural networks," Int. J. Circuit Theory and Applications, vol 20, pp 453-468, Sept 1992.

[8] C. Toumazou, F.J. Lidgey and D. Haigh, Analogue IC design : the Current-mode Approach, IEEE 1990.

[9] B. Mirzai, D Lim, and G. S. Moschytz, "Robust CNN templates: theory and simulations", Proc. IEEE 4th Int. Workshop on CNN and Applications (CNNA), Spain, pp 393-398, June, 1996.

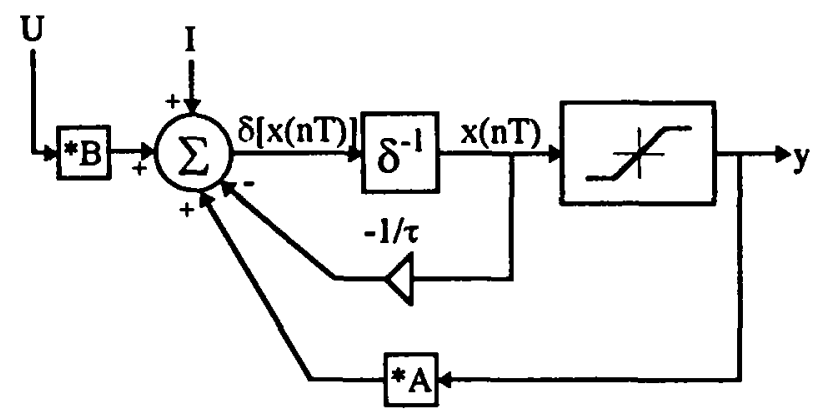

Fig.1 A block diagram representation of the standard CNN with $\delta^{-1}$ as a dynamic element. Note that $\delta^{-1}$ can be realised with a switched capacitor circuit or switched current circuit. 